University of Texas Rio Grande Valley

ScholarWorks @ UTRGV

Chemistry Faculty Publications and

Presentations

College of Sciences

$10-27-2018$

\title{
Molten-Salt Synthesis of Complex Metal Oxide Nanoparticles
}

Jose P. Zuniga

Maya Abdou

Santosh K. Gupta

Yuanbing Mao

The University of Texas Rio Grande Valley

Follow this and additional works at: https://scholarworks.utrgv.edu/chem_fac

Part of the Chemistry Commons

\section{Recommended Citation}

Zuniga, J. P., Abdou, M., Gupta, S. K., \& Mao, Y. (2018). Molten-Salt Synthesis of Complex Metal Oxide Nanoparticles. JoVE (Journal of Visualized Experiments), 140, e58482. https://doi.org/10.3791/58482

This Article is brought to you for free and open access by the College of Sciences at ScholarWorks @ UTRGV. It has been accepted for inclusion in Chemistry Faculty Publications and Presentations by an authorized administrator of ScholarWorks@ UTRGV. For more information, please contact justin.white@utrgv.edu,william.flores01@utrgv.edu. 


\title{
Video Article
}

\section{Molten-Salt Synthesis of Complex Metal Oxide Nanoparticles}

\author{
Jose P. Zuniga ${ }^{1}$, Maya Abdou ${ }^{1}$, Santosh K. Gupta ${ }^{1,2}$, Yuanbing Mao ${ }^{1,3}$ \\ ${ }^{1}$ Department of Chemistry, University of Texas Rio Grande Valley \\ ${ }^{2}$ Radiochemistry Division, Bhabha Atomic Research Centre \\ ${ }^{3}$ School of Earth, Environmental, and Marine Sciences, University of Texas Rio Grande Valley
}

Correspondence to: Yuanbing Mao at yuanbing.mao@utrgv.edu

URL: https://www.jove.com/video/58482

DOI: doi:10.3791/58482

Keywords: Coprecipitation, molten-salt synthesis, complex metal oxides, lanthanum hafnium oxide, nanoparticles, precursor

Date Published: $8 / 13 / 2018$

Citation: Zuniga, J.P., Abdou, M., Gupta, S.K., Mao, Y. Molten-Salt Synthesis of Complex Metal Oxide Nanoparticles. J. Vis. Exp. (), e58482, doi:10.3791/58482 (2018).

\section{Abstract}

The development of feasible synthesis methods is critical for the successful exploration of novel properties and potential applications of nanomaterials. Here, we introduce the molten-salt synthesis (MSS) method for making metal oxide nanomaterials. Advantages over other methods include its simplicity, greenness, reliability, scalability, and generalizability. Using pyrochlore lanthanum hafnium oxide $\left(\mathrm{La}_{2} \mathrm{Hf}_{2} \mathrm{O}_{7}\right)$ as a representative, we describe the MSS protocol for the successful synthesis of complex metal oxide nanoparticles (NPs). Furthermore, this method has the unique ability to produce NPs with different material features by changing various synthesis parameters such as $\mathrm{pH}$, temperature, duration, and post-annealing. By fine-tuning these parameters, we are able to synthesize highly uniform, non-agglomerated, and highly crystalline NPs. As a specific example, we vary the particle size of the $\mathrm{La}_{2} \mathrm{Hf}_{2} \mathrm{O}_{7} \mathrm{NPs}$ by changing the concentration of the ammonium hydroxide solution used in the MSS process, which allows us to further explore the effect of particle size on various properties. It is expected that the MSS method will become a more popular synthesis method for nanomaterials and more widely employed in the nanoscience and nanotechnology community in the upcoming years.

\section{Video Link}

The video component of this article can be found at https://www.jove.com/video/58482/

\section{Introduction}

Molten-salt synthesis (MSS) involves the use of a molten salt as the reaction medium for preparing nanomaterials from their constituent precursors. The molten salt acts as the solvent and facilitates the enhanced reaction rate by increasing the contact area between reactants and their mobility. The choice of molten salts is of paramount importance for the success of the MSS method. The salt must meet some important quality requirements such as low melting point, compatibility with reacting species, and optimum aqueous solubility. Molten salt has been used previously to enhance the rate of solid-state reactions; however, in a flux system, only a small amount of molten salt is used (unlike in MSS, in which a large quantity is added to form a soluble medium for the reaction and control the properties of the synthesized nanomaterials, such as particle size, shape, and crystallinity, etc.). In this sense, MSS is a modification of the powder metallurgical method and different from the flux method $^{1,2,3}$. The employment of molten salt can (1) increase reaction kinetic rate ${ }^{4}$ while decreasing synthesis temperature ${ }^{5},(2)$ increase the degree of reactant homogeneity ${ }^{6},(3)$ control crystalline size and morphology ${ }^{7}$, and (4) reduce the level of agglomeration.

Nanomaterials have been in high demand in scientific research and novel industrial applications because of their superior electrical, chemical, magnetic, optical, electronic, and thermal properties. Their properties are highly dependent on the particle size, shape, and crystallinity. Compared with other synthesis methods for nanomaterials, MSS has several obvious advantages; although, it is not yet as well-known as other synthesis methods in the nanoscience and nanotechnology community. As described below, these advantages include its simplicity, reliability, scalability, generalizability, environmental friendliness, cost effectiveness, relative low synthesis temperature, and free agglomeration of NPs with clean surface ${ }^{8}$.

Simplicity: The MSS process can be easily carried out in a simple laboratory with basic facilities. No sophisticated instrumentation is needed. Precursors and molten salts are air stable with no need for glove box handling.

Reliability: Once all initial synthesis parameters such as concentration, $\mathrm{pH}$, processing time, and annealing temperature are optimized, highquality and pure products are assured when using the MSS method. If all synthesis steps are carried out properly, the final products may attain all basic criteria needed for good-quality NPs. A novice to the MSS method will not change the synthesis outcome, as long as all synthesis parameters are properly and carefully followed.

Scalability: The MSS method's ability to produce large quantities of size- and shape-controlled particles is crucial. This critical factor is important because it allows for the determination of industrial usefulness and efficiency. Compared to other synthesis techniques, MSS can easily generate 
a sufficient amount of products by adjusting stoichiometric amounts during the process. This is an important feature of the method because it allows for convenience at the industrial level, making it a more desired approach due to this scalability ${ }^{9,10}$.

Generalizability: The MSS method is also a generalizable technique to produce nanoparticles with various compositions. Other than simple metal oxides and some fluorides, nanomaterials of complex metal oxides that have been successfully synthesized by the MSS method include perovskites $\left(\mathrm{ABO}_{3}\right)^{10,11,12,13,14}$, spinel $\left(\mathrm{AB}_{2} \mathrm{O}_{4}\right)^{15,16}$, pyrochlore $\left(\mathrm{A}_{2} \mathrm{~B}_{2} \mathrm{O}_{7}\right)^{4,17,18,19}$, and orthorhombicstructures $\left(\mathrm{A}_{2} \mathrm{~B}_{4} \mathrm{O}_{9}\right)^{2,3,20}$. More specifically, these nanomaterials include ferrites, titanates, niobates, mullite, aluminium borate, wollastonite, and carbonated apatite ${ }^{7,9,21}$. The MSS method has also been used to produce nanomaterials of various morphologies such as nanospheres ${ }^{4}$, ceramics powder bodies ${ }^{22}$, nanoflakes ${ }^{23}$, nanoplates ${ }^{7}$, nanorods ${ }^{24}$, and core-shell nanoparticles (NPs) ${ }^{25}$, depending on synthesis conditions and crystal structure of the products.

Environmental friendliness: Several traditional methods for making nanomaterials involve the use of large amounts of organic solvents and toxic agents that generate environmental issues. The partial or total elimination of the use of them and the generation of waste by sustainable processes is in demand of green chemistry nowadays. The MSS method is an environmentally friendly approach to synthesize nanomaterials by employing nontoxic chemical and renewable materials and minimizing waste, byproducts, and energy.

Relative low synthesis temperature: The processing temperature of the MSS method is relatively low compared to that required in a conventional solid-state reaction ${ }^{26}$ or a sol-gel combustion reaction ${ }^{27}$. This lower temperature saves energy while producing high-quality NPs.

Cost effectiveness: The MSS method does not require any harsh or costly reactants or solvents nor any specialized instrumentation. Water is the main solvent used for washing away the used molten salts, which are also cheap. Moreover, experimental setup needed includes only simple glassware and a furnace without specialized instrumentation, while nanomaterials with complex composition and refractory nature can be produced.

Agglomeration free with clean surface: During the MSS process, the formed nanoparticles are well-dispersed in the molten salt medium due to its large quantity, used along with its high ionic strength and viscosity ${ }^{1,6,8}$. Unlike colloidal synthesis and most hydrothermal/solvothermal processes, no protective surface layer is necessary to prevent the continuous growth and agglomeration of the formed NPs.

Exemplary synthesis of complex metal oxide NPs by the MSS method: The MSS method as a universal and cost-effective approach to rationally and large-scale synthesize nanomaterials for a sufficiently wide spectrum of material may be highly welcomed by scientists working in nanoscience and nanotechnology. Here, lanthanum hafnate $\left(\mathrm{La}_{2} \mathrm{Hf}_{2} \mathrm{O}_{7}\right)$ was selected because of its multifunctional applications in the areas of $X$ ray imaging, high $k$-dielectric, luminescence, thermographic phosphor, thermal barrier coating, and nuclear waste host. $\mathrm{La}_{2} \mathrm{Hf}_{2} \mathrm{O}_{7}$ is also a good host for doped scintillators due to its high density, large effective atomic number, and the possibility of its crystal structure to be engineered along with an order-disorder phase transition. It belongs to the $\mathrm{A}_{2} \mathrm{~B}_{2} \mathrm{O}_{7}$ family of compounds, in which " $\mathrm{A}$ " is a rare-earth element with a +3 oxidation state, and "B" represents a transition metallic element with $\mathrm{a}+4$ oxidation state. However, due to the refractory nature and complex chemical composition, there has been a lack of proper low-temperature and large-scale synthesis methods for $\mathrm{La}_{2} \mathrm{Hf}_{2} \mathrm{O}_{7} \mathrm{NPs}$.

For fundamental scientific investigation and advanced technological applications, it is a prerequisite to make monodisperse, high-quality, and uniform $\mathrm{A}_{2} \mathrm{~B}_{2} \mathrm{O}_{7} \mathrm{NPs}$. Here we use the synthesis of highly crystalline $\mathrm{La}_{2} \mathrm{Hf}_{2} \mathrm{O}_{7} \mathrm{NPs}$ as an example to demonstrate the advantages of the MSS method. As schematically shown in Figure 1, $\mathrm{La}_{2} \mathrm{Hf}_{2} \mathrm{O}_{7} \mathrm{NPs}$ were prepared by the MSS method with a two-step process following our previous reports. First, a single-source complex precursor of $\mathrm{La}(\mathrm{OH})_{3} \cdot \mathrm{HfO}(\mathrm{OH})_{2} \cdot n \mathrm{H}_{2} \mathrm{O}$ was prepared via a coprecipitation route. In the second step, sizecontrollable $\mathrm{La}_{2} \mathrm{Hf}_{2} \mathrm{O}_{7} \mathrm{NPs}$ were synthesized through the facile MSS process using the single-source complex precursor and nitrate mixture $\left(\mathrm{NaNO}_{3}: \mathrm{KNO}_{3}=1: 1\right.$, molar ratio) at $650^{\circ} \mathrm{C}$ for $6 \mathrm{~h}$.

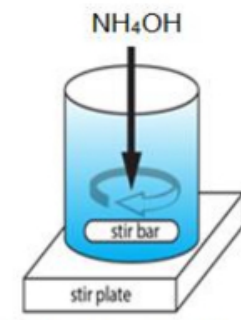

$\mathrm{La}\left(\mathrm{NO}_{3}\right)_{3}+\mathrm{HfOCl}_{2}$

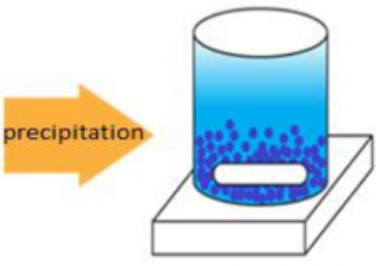

$\mathrm{La}(\mathrm{OH})_{3} \cdot \mathrm{HfO}\left(\mathrm{OH}_{2}\right) \cdot n\left(\mathrm{H}_{2} \mathrm{O}\right)$

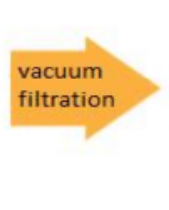

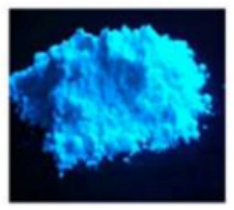

Precursor

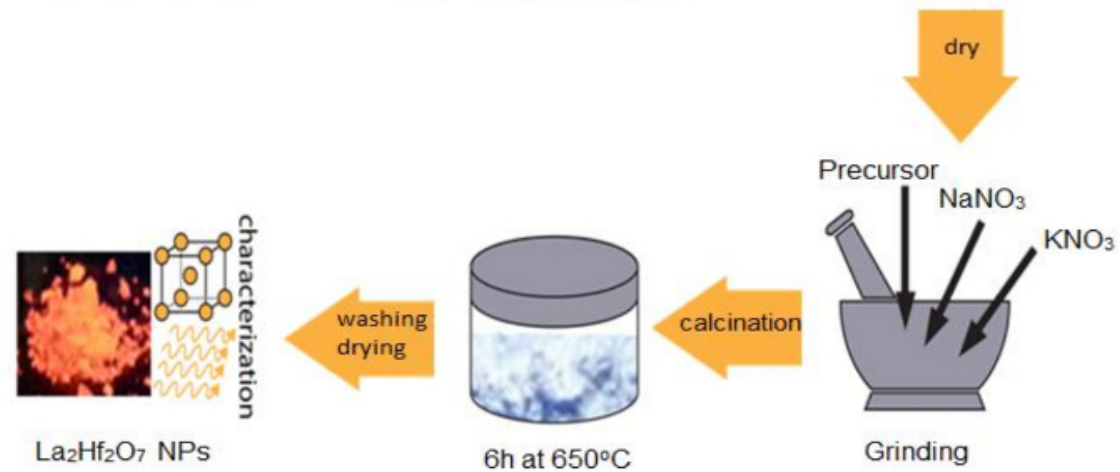

Figure 1: Schematic of the synthesis steps for $\mathrm{La}_{2} \mathrm{Hf}_{2} \mathrm{O}_{7} \mathrm{NPs}$ via the MSS method. Please click here to view a larger version of this figure. 


\section{Protocol}

\section{Preparation of a Single-Source Complex Precursor via a Coprecipitation Route}

1. Preparation of lanthanum and hafnium precursor solution

1. Measure $200 \mathrm{~mL}$ of distilled water in a $500 \mathrm{~mL}$ beaker and start stirring at $300 \mathrm{rpm}$.

2. Dissolve lanthanum and hafnium precursors in the stirring water [i.e., $2.165 \mathrm{~g}$ of lanthanum nitrate hexahydrate $\left(\mathrm{La}\left(\mathrm{NO}_{3}\right)_{3} \cdot 6 \mathrm{H}_{2} \mathrm{O}\right)$ and $2.0476 \mathrm{~g}$ of hafnium dichloride oxide octahydrate $\left.\left(\mathrm{HfOCl}_{2} \cdot 8 \mathrm{H}_{2} \mathrm{O}\right)\right]$.

3. Let the solution stir for $30 \mathrm{~min}$ before starting the titration.

2. Preparation of diluted ammonia solution

1. Prepare $200 \mathrm{~mL}$ of diluted ammonia solution with different concentrations, including $0.75 \%, 1.5 \%, 3.0 \%, 6.0 \%$, and $7.5 \%$. For example, add $20 \mathrm{~mL}$ of concentrated ammonia solution $\left(\mathrm{NH}_{4} \mathrm{OH} 28-30 \%\right)$ to $180 \mathrm{~mL}$ of distilled water in a separate beaker to make $3.0 \%$ diluted ammonia solution.

3. Titration and washing the single source complex precursor

1. Add the diluted ammonia solution prepared in the previous step into a burette and ensure that the burette is covered at all times, since ammonia solution tends to evaporate which decreases its concentration.

2. Add the diluted ammonia solution in the burette into the stirring solution of lanthanum nitrate and hafnium dichloride oxide dropwise.

3. Adjust the dropping speed of the ammonia solution accordingly so that it will be added over a period of $2 \mathrm{~h}$.

4. After several $\mathrm{mL}$ of ammonia solution have been delivered, ensure that the solution becomes cloudy. This is a simple sign that the precipitate of the single-source complex precursor of $\mathrm{La}(\mathrm{OH})_{3} \cdot \mathrm{HfO}(\mathrm{OH})_{2} \cdot n \mathrm{H}_{2} \mathrm{O}$ is forming.

5. After $2 \mathrm{~h}$, remove the stirring bar and allow the precipitate to age overnight.

6. Check the $\mathrm{pH}$ of the coprecipitated solution before washing. Wash the precipitate with distilled water until the supernatant reaches a neutral $\mathrm{pH}$, which normally takes 5-8 washes.

4. Vacuum filtration and drying of precursor

1. Vacuum filter the coprecipitated solution using a filter paper with a coarse porosity $(40-60 \mu \mathrm{m}$; see Table of Materials) to separate the solid precipitate from the supernatant.

2. Ensure that all complex precursor remnants are washed from the walls of the beaker.

3. Air dry the resulting single-source complex precursor $\mathrm{La}(\mathrm{OH})_{3} \cdot \mathrm{HfO}(\mathrm{OH})_{2} \cdot n \mathrm{H}_{2} \mathrm{O}$ at room temperature overnight.

\section{Molten-Salt Synthesis of Lanthanum Hafnate NPs}

1. Preparation of salt and precursor mixture

1. Measure $30 \mathrm{mmol}(3.033 \mathrm{~g})$ of potassium nitrate $\left(\mathrm{KNO}_{3}\right)$ and $30 \mathrm{mmol}(2.549 \mathrm{~g})$ of sodium nitrate $\left(\mathrm{NaNO}_{3}\right)$.

2. Combine the measured salts with $0.35 \mathrm{~g}$ of the as-prepared single-source complex precursor $\mathrm{La}(\mathrm{OH})_{3} \cdot \mathrm{HfO}(\mathrm{OH})_{2} \cdot n \mathrm{H}_{2} \mathrm{O}$.

3. If necessary, add $1-5 \mathrm{~mL}$ of acetone or ethanol to the mixture to facilitate the grinding. Ensure that all the solvent is evaporated before placing the mixture into a crucible.

4. Grind the mixed salts and precursor as fine as possible for about 30 min using a mortar and pestle.

2. Molten-salt processing

1. Place the resulting mixture in a corundum crucible, then place it in a muffle furnace.

2. Set the furnace at $650{ }^{\circ} \mathrm{C}$ for $6 \mathrm{~h}$ with a ramp rate of $10^{\circ} \mathrm{C} / \mathrm{min}$.

3. After the sample and furnace have cooled to room temperature, take out the crucible and soak the sample in a beaker filled with distilled water overnight.

3. Washing and drying the $\mathrm{La}_{2} \mathrm{Hf}_{2} \mathrm{O}_{7} \mathrm{NPs}$

1. Empty the sample from the crucible into a $1 \mathrm{~L}$ beaker.

2. Wash the sample with distilled water 5-8 times until the supernatant is clear of salts and not cloudy anymore.

3. Purify the product by centrifugation or vacuum filtration to remove any residual impurity.

4. Dry the product in the oven at $90{ }^{\circ} \mathrm{C}$ overnight.

\section{Representative Results}

The as-synthesized $\mathrm{La}_{2} \mathrm{Hf}_{2} \mathrm{O}_{7} \mathrm{NPs}$ may exist in the ordered pyrochlore phase. However, chemical doping, pressure, and temperature could modify the phase to defect fluorite. It is possible for our material to have multiple phases; however, here we focus only on the pyrochlore phase for simplicity. X-ray diffraction (XRD) and Raman spectroscopy have been used to systematically characterize their phase purity, structure, and phase. The crystalline size can be calculated using Debye-Scherrer's formula. On the other hand, Raman spectroscopy is used as a secondary structural characterization technique due to it high sensitivity to $\mathrm{M}-\mathrm{O}$ vibrational modes ${ }^{28}$. It identifies the exact crystallographic phase of the $\mathrm{La}_{2} \mathrm{Hf}_{2} \mathrm{O}_{7} \mathrm{NPs}^{29}$. 
Compared to other synthetic techniques such as solid-state reactions, MSS has a high reaction constant, meaning that the reaction is more likely to go in the forward direction ${ }^{30}$. The production of pyrochlore $\mathrm{La}_{2} \mathrm{Hf}_{2} \mathrm{O}_{7}$ is achieved by increasing the contact interfacial area and mobility of the reactant species inside the molten salt system. Figure 2 illustrates the XRD patterns of the synthesized $\mathrm{La}_{2} \mathrm{Hf}_{2} \mathrm{O}_{7} \mathrm{NPs}_{\mathrm{S}}$ made by the MSS method from the single-source complex precursors using different concentrations of ammonia solution. XRD results shows the formation of pure $\mathrm{La}_{2} \mathrm{Hf}_{2} \mathrm{O}_{7}$ with high crystallinity. The peak position and Miller indices may be indexed based on the Joint Committee on Powder Diffraction Standard (JCPDS) pattern \#78-1292. No visible impurity phases such as $\mathrm{La}_{2} \mathrm{O}_{3}, \mathrm{Eu}_{2} \mathrm{O}_{3}$, or $\mathrm{HfO}_{2}$ were detected. There is no change in XRD pattern as a function of the used ammonia concentration except for the increase of particle size. Table 1 shows the corresponding calculated crystal parameter and particle size of the $\mathrm{La}_{2} \mathrm{Hf}_{2} \mathrm{O}_{7} \mathrm{NPs}$.

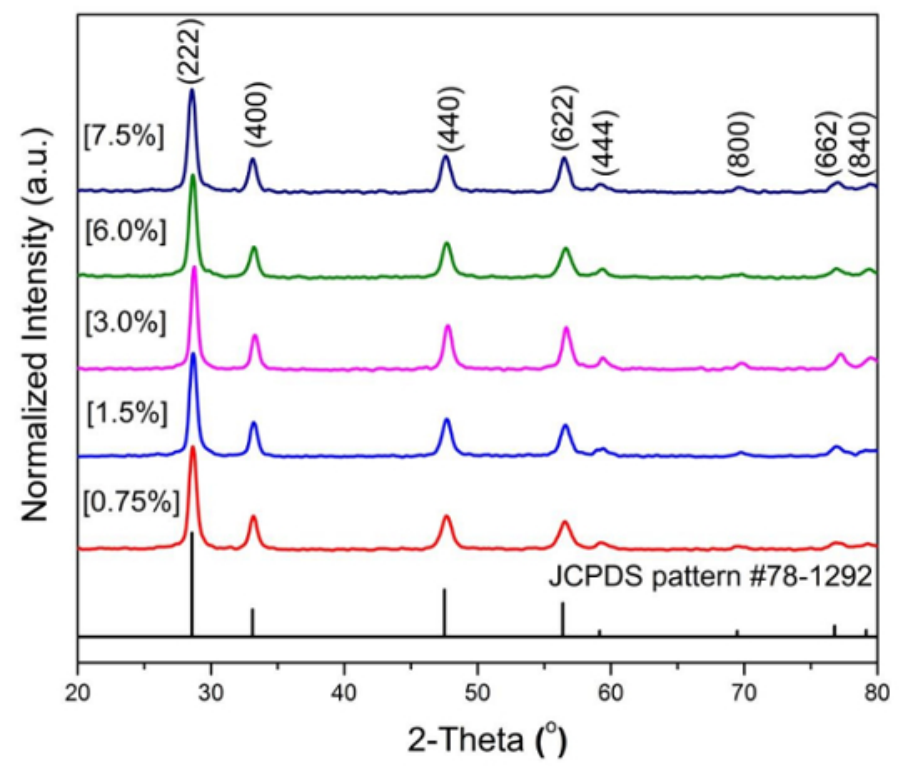

Figure 2: X-ray diffraction patterns of the $\mathrm{La}_{2} \mathrm{Hf}_{2} \mathrm{O}_{7} \mathrm{NPs}$ prepared by adjusting the used ammonia solution concentration then indexed based on the defect fluorite pattern. Please click here to view a larger version of this figure.

\begin{tabular}{|c|c|c|c|c|}
\hline \multirow{2}{*}{$\begin{array}{l}\text { Concentration of } \\
\mathrm{NH}_{4} \mathrm{OH}(\mathrm{aq}) \text { used }\end{array}$} & \multicolumn{4}{|c|}{ XRD data of the synthesized $\mathrm{La}_{2} \mathrm{Hf}_{2} \mathrm{O}_{7} \mathrm{NPs}$} \\
\hline & $2 \theta\left({ }^{\circ}\right)$ & FWHM $(\beta)$ & Lattice parameters $(\AA)$ & Particle size (nm) \\
\hline$[0.75 \%]$ & 28.57 & 0.4 & 10.81 & 19.00 \\
\hline [1.5\%] & 28.64 & 0.39 & 10.79 & 20.00 \\
\hline [3.0\%] & 28.67 & 0.37 & 10.78 & 21.00 \\
\hline [6.0\%] & 28.69 & 0.31 & 10.77 & 26.00 \\
\hline [7.5\%] & 28.74 & 0.27 & 10.75 & 29.00 \\
\hline
\end{tabular}

Table 1: Crystallographic parameter and particle size of the $\mathrm{La}_{2} \mathrm{Hf}_{2} \mathrm{O}_{7} \mathrm{NPs}$ prepared by adjusting the used ammonia solution concentration showing a continuous particle size growth. FWHM = full-width at half-maximum.

There are two common phases of the $\mathrm{A}_{2} \mathrm{~B}_{2} \mathrm{O}_{7}$ compounds:the disordered fluorite phase and ordered pyrochlore phase. The disordered fluorite phase exists in the $\mathrm{Fm}^{-} \mathrm{m}$ space group, wherein all the cationic ions $\left(\mathrm{A}^{3+}\right.$ and $\left.\mathrm{B}^{4+}\right)$ are randomly distributed, allowing for one active mode. On the other hand, ordered pyrochlore phase exists in $\mathrm{F} \overline{\mathrm{d}} \mathrm{m}$ space group. Therefore, it exhibits close structural resemblance to fluorite phase except that there are two cationic sites, three anionic sites $\left[48 \mathrm{f}\left(\mathrm{O}_{\mathrm{I}}\right), 8 \mathrm{a}\left(\mathrm{O}_{\mathrm{II}}\right)\right.$, and $\left.8 \mathrm{~b}\left(\mathrm{O}_{\mathrm{III}}\right)\right]$, and $1 / 8^{\text {th }}$ of the oxygen ions $\left(\mathrm{O}_{\mathrm{III}}\right)$ at $8 \mathrm{~b}$ site absent in the pyrochlore structure, allowing for 6 active modes ${ }^{19,28,29}$. Based on the group theory, the defect fluorite structure has one vibrational mode due to $\mathrm{T}_{2 \mathrm{~g}}$, while a pyrochlore phase has six vibrational modes in the range of $200-1000 \mathrm{~cm}^{-1}$. This information is vital for the correct phase identification of the $\mathrm{La}_{2} \mathrm{Hf}_{2} \mathrm{O}_{7} \mathrm{NPs}$ (Figure 3). In this case, the prepared $\mathrm{La}_{2} \mathrm{Hf}_{2} \mathrm{O}_{7} \mathrm{NPs}$ are in pure pyrochlore form. 


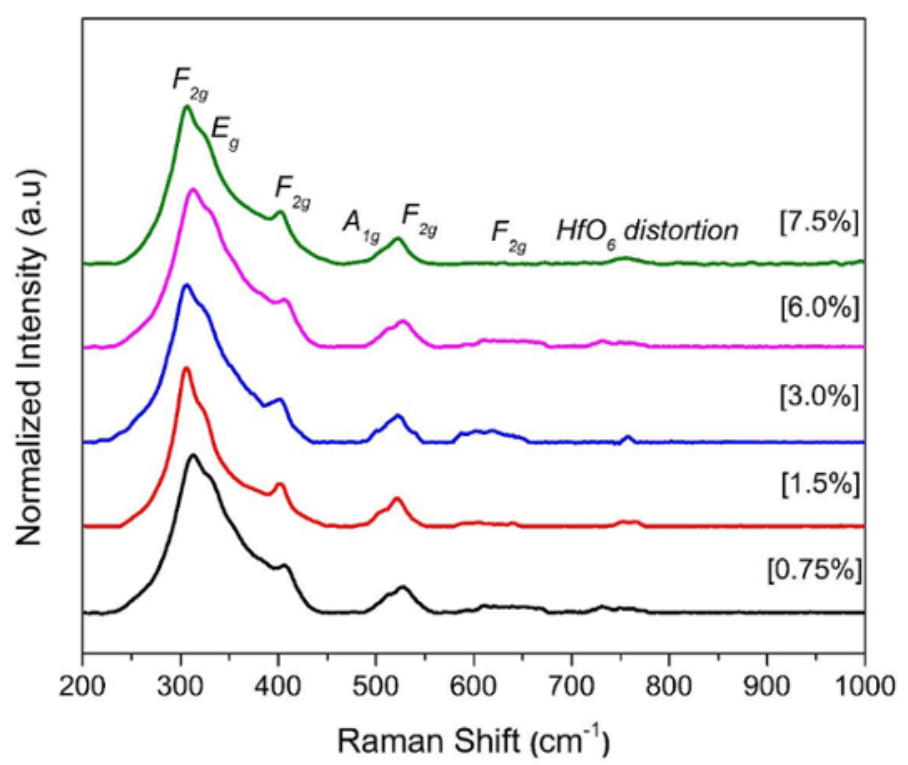

Figure 3: Raman spectra of the $\mathrm{La}_{2} \mathrm{Hf}_{2} \mathrm{O}_{7} \mathrm{NPs}$ prepared by adjusting the used ammonia solution concentration, with six active modes pertaining to the pyrochlore phase. Please click here to view a larger version of this figure.

\section{Discussion}

The chart in Figure 4 provides several reliable controlling factors of the MSS method and accounts for alternative pathways to fine-tune the features of synthesized nanomaterials. In addition, it helps identify critical steps in the MSS process.

\section{Synthesis steps Controlling factors}

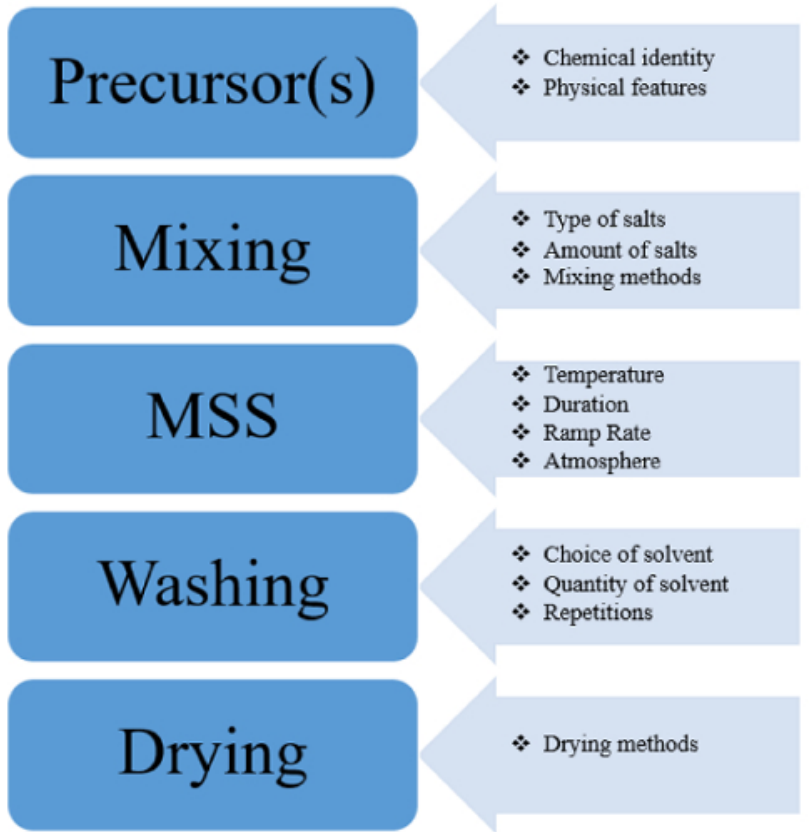

Figure 4: Flowchart of the critical steps of MSS indicating the controlling factors of the synthetic procedure for NPs with potential pathways to fine-tune their characteristics. Please click here to view a larger version of this figure.

First, the identity of precursors is critical, especially for the synthesis of complex metal oxide NPs. When producing $A_{2} B_{2} \mathrm{O}_{7} N P s$, it is important to first generate a single-source complex precursor that contains the main elements making up the final product [i.e., $\mathrm{A}$ and $\mathrm{B}$, at an atomic level with the formula of $\mathrm{A}(\mathrm{OH})_{3} \cdot \mathrm{BO}(\mathrm{OH})_{2} \cdot n \mathrm{H}_{2} \mathrm{O}$ ]. In this experiment, the particle size of the final $\mathrm{La}_{2} \mathrm{Hf}_{2} \mathrm{O}_{7} \mathrm{NPs}$ could be controlled during this step by adjusting the concentration of the titrant ammonia solution, where a higher ammonia solution concentration generated bigger $\mathrm{La}_{2} \mathrm{Hf}_{2} \mathrm{O}_{7} \mathrm{NPs}$. Other trials have also been performed to make $\mathrm{A}_{2} \mathrm{~B}_{2} \mathrm{O}_{7} \mathrm{NPs}$ using different precursors, such as commercially available nitrates or oxides containing the main elements of $\mathrm{A}_{2} \mathrm{~B}_{2} \mathrm{O}_{7}$; however, none have worked ${ }^{4}$. The advantages of this amorphous single-source complex precursor 
are that the composing elements of $\mathrm{A}_{2} \mathrm{~B}_{2} \mathrm{O}_{7}$ are mixed at an atomic level and are homogeneously distributed in the same order as the final $\mathrm{A}_{2} \mathrm{~B}_{2} \mathrm{O}_{7}$ product, decreasing the space or range the reactants that need to be transported.

Second, the selection of proper molten salt has major importance in obtaining nanomaterials with desirable morphology and characteristics. The melting point of the selected salt should be appropriate for the synthesis of the required phase of nanomaterials. The formation of nanomaterials is ultimately governed by the melting point of salts used as molten solvents, such as alkali chloride, sulfate, carbonate, or hydroxide, $1,6,31,32$. It has been reported that the use of different molten salts can modify the formation temperature, thereby altering the particle size and morphology ${ }^{18}$.

In addition, the ramp rate tends to affect the synthesis of the NPs. A fast ramp rate tends to generate agglomerated NPs ${ }^{33}$, which affects their properties. In the case of luminescent and scintillation materials, high agglomeration is not desirable, as it can scatter excited and emitted light ${ }^{17,19}$. A slow ramp rate is thus more suitable and allows enough time to reach the actual temperature inside the crucible. A slower cooling process usually allows for NPs with a spherical shape to form ${ }^{34}$.

Another important criterion for the selection of the salt is that it should have sufficient aqueous solubility. With simple washing by water, the used molten salt should be easily washed away after the MSS.

Finally, the addition of a volatile liquid such as acetone and ethanol during the mixing of the salts used and single-source complex precursor helps ease the grinding process but is not essential. The addition of a liquid is helpful to make a homogenous mixture in a shorter time and requires less effort, which is important in generating pure products. The added volatile liquid does not affect the characteristics of the resultant NPs since it fully evaporates by the end of the grinding process. Due to its high volatility, the amount added may range anywhere from 1 to $5 \mathrm{~mL}$.

The MSS method is a straightforward and simple way to synthesize NPs at any university and industrial location. However, modifications of the protocol can be made. For example, in case a vacuum filter system is not available, the single-source complex precursor obtained from the coprecipitation step can be centrifuged. Different eutectic salt mixtures can be used to engineer NPs with desired characteristics.

Even though the MSS method is generally easy to be employed, limitations include (1) the possibility of the product formation being limited by the contact area of the dissimilar reactants in the reaction ${ }^{1}$. Additionally, (2) not every nanostructured product can be formed within selected molten salts. These cases are rare but can certainly happen ${ }^{35}$. It is desirable that the selected salts act only as pure solvents and do not react with the reactants or products. Furthermore, common mistakes that may hinder the quality of the final nanoparticle products include the following: (1) First, if the burette is not covered during the titration, this may change the concentration of the ammonium hydroxide and eventually change the size of obtained nanoparticles. Another common mistake is (2) not giving the coprecipitation a period of two hours to form. Adding the ammonia titrant too fast affects the coprecipitation kinetics, which might render inhomogeneous complex precursor. The third pitfall is (3) not grinding the salt(s) and precursor(s) as fine as possible, which generates impure products or inhomogeneous particles.

The MSS method is simple, effective, fast, low-temperature, and cost-efficient as proven with previously reported results, compared to conventional solid-state and sol-gel/combustion synthesis methods ${ }^{27}$. It is also scalable, reliable, and generalizable in regards to making agglomeration-free NPs without a surface protective layer, unlike colloidal and hydrothermal/solvothermal synthesis methods.

Application of the MSS method has widely spread in the last couple of decades, from ferroelectric and ferromagnetic materials to materials for Li-ion batteries ${ }^{36}$, semiconductors ${ }^{17,37}$, phosphors ${ }^{17,19}$, and electro-catalysts ${ }^{38,39}$, mainly for nanosized materials, especially those with complex compositions. In conclusion, the MSS method provides a suitable pathway in the synthesis of simple and complex metal oxide NPs. It is expected that the MSS method will become an even more popular synthesis method for nanomaterials and more widely employed in the nanoscience and nanotechnology community in the upcoming years.

\section{Disclosures}

There are no conflicts to declare.

\section{Acknowledgements}

The authors thank the financial support provided by the National Science Foundation under CHE (award \#1710160) and the USDA National Institute of Food and Agriculture (award \#2015-38422-24059). The Department of Chemistry at the University of Texas Rio Grande Valley is grateful for the generous support provided by a Departmental Grant from the Robert A. Welch Foundation (Grant No. BX-0048). S.K.G. would like to thank the United States-India Education Foundation (USIEF) and International Institute of Education (IIE) for his Fulbright Nehru Postdoctoral Fellowship (award \#2268/FNPDR/2017).

\section{References}

1. Kimura, T. Molten Salt Synthesis of Ceramic Powders. Advances in Ceramics. InTech. Chapter $4,75-100,(2011)$.

2. Mao, Y., Park, T.-J., Wong, S. S. Synthesis of classes of ternary metal oxide nanostructures. Chemical Communications. 0 , (46), $5721-5735$ (2005).

3. Mao, Y., Zhou, H., Wong, S. S. Perovskite-phase metal oxide nanostructures: synthesis, properties, and applications. Material Matters. 5 ,50-53 (2010).

4. Mao, Y., Guo, X., Huang, J. Y., Wang, K. L., Chang, J. P. Luminescent Nanocrystals with $\mathrm{A}_{2} \mathrm{~B}_{2} \mathrm{O}_{7}$ Composition Synthesized by a Kinetically Modified Molten Salt Method. The Journal of Physical Chemistry C. 113 , (4), 1204-1208 (2009).

5. Yu, Y., Wang, S., Li, W., Chen, Z. Low temperature synthesis of $\mathrm{LaB}_{6}$ nanoparticles by a molten salt route Powder Technology. 323,203-207 (2018). 
6. Liu, X., Fechler, N., Antonietti, M. Salt melt synthesis of ceramics, semiconductors and carbon nanostructures. Chemical Society Reviews. 42 , (21), 8237-8265 (2013).

7. Chang, Y., Wu, J., Zhang, M., Kupp, E., Messing, C. L. Molten salt synthesis of morphology controlled alpha-alumina platelets Ceramics International. 43 , (15), 12684-12688 (2017).

8. Mao, Y., Park, T.-J., Zhang, F., Zhou, H., Wong, S. S. Environmentally friendly methodologies for nanostructure synthesis. Small. 3 , (7), $1122-1139$ (2007).

9. Liu, J. R., Hong, R. Y., Feng, W. G., Badami, D., Wang, Y. Q. Large scale production of strontium ferrite by molten salt assited coprecipitation. Powder Technology. $262,142-149$ (2014).

10. Yuanbing, M., Banerjee, S., Wong, S.S. Large-scale synthesis of single-crystalline perovskite nanostructures. Journal of the American Chemical Society. $125,(51), 15718-15719$ (2003).

11. Mao, Y. Facile synthesis of ferromagnetic double perovskite oxide $\mathrm{La}_{2} \mathrm{BMnO}_{6}$ nanoparticles. RSC Advances. 2 , (33), 12675-12678 (2012).

12. Hailili, R., Wang, C., Lichtfouse, E. Perovskite nanostructures assembled in molten salt based on halogen anions $\mathrm{KX}(\mathrm{X}=\mathrm{F}, \mathrm{Cl}$ and $\mathrm{Br})$ : Regulated morphology and defect-mediated photocatalytic activity. Applied Catalysis B: Enviromental. 232 ,531-543 (2018).

13. Yuanbing Mao, J. P., John S. McCloy. Magnetic properties of double perovskite oxide $\mathrm{La}_{2} \mathrm{BMnO}_{6}$ nanocrystals. Nanoscale. 5 , (11), $4720-4728$ (2013).

14. Mao, Y., Wong, S. S. Reproducible composition and shape control of crystalline $\mathrm{Ca}_{1-\mathrm{x}} \mathrm{Sr}_{\mathrm{x}} \mathrm{TiO}_{3}$ perovskite nanoparticles. Advanced Materials. $17,(18), 2194-2199(2005)$.

15. Rojas-Hernandez, R. E., et al. Original Synthetic Route To Obtain a $\mathrm{SrAl}_{2} \mathrm{O}_{4}$ Phosphor by the Molten Salt Method: Insights into the Reaction Mechanism and Enhancement of the Persistent Luminescence. Inorganic Chemistry. 54 , (20), 9896-9907 (2015).

16. Reddy, M. V., Xu, Y., Rajarajan, V., Ouyang, T., Chowdari, B. V. R. Template Free Facile Molten Synthesis and Energy Storage Studies on $\mathrm{MCo}_{2} \mathrm{O}_{4}(\mathrm{M}=\mathrm{Mg}, \mathrm{Mn})$ as Anode for Li-lon Batteries. ACS Sustainable Chemistry and Engineering. 3 , (12), 3035-3042 (2015).

17. Zuniga, J. P., Gupta, S. K., Pokhrel, M., Mao, Y. Exploring optical properties of $\mathrm{La}_{2} \mathrm{Hf}_{2} \mathrm{O}_{7}: \mathrm{Pr}^{3+}$ nanoparticles under UV and X-ray excitations for potential lighting and scintillating applications. New Journal of Chemistry. 42 , (12), 9381-9392 (2018).

18. Pokhrel, M., Wahid, K., Mao, Y. Systematic Studies on $\mathrm{RE}_{2} \mathrm{Hf}_{2} \mathrm{O}_{7}: 5 \% \mathrm{Eu}^{3+}$ (RE = Y, La, Pr, Gd, Er, and Lu) Nanoparticles: Effects of the A-Site $\mathrm{RE}^{3+}$ Cation and Calcination on Structure and Photoluminescence. The Journal of Physical Chemistry C. 120, (27), 14828-14839 (2016).

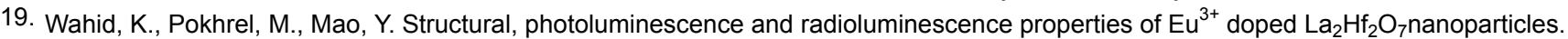
Journal of Solid State Chemistry. $245,89-97$ (2017).

20. Park, T.-J., Papaefthymiou, G. C., Moodenbaugh, A. R., Mao, Y., Wong, S. S. Synthesis and characterization of submicron single-crystalline $\mathrm{Bi}_{2} \mathrm{Fe}_{4} \mathrm{O}_{9}$ cubes. Journal of Materials Chemistry. 15 , (21), 2099-2105 (2005).

21. Gilbert, M. R. Molten salt synthesis of titanate pyrochlore waste-forms. Ceramics International. 42 , (4), $5263-5270$ (2016).

22. Huang, Z., et al. Molten salt synthesis of $\mathrm{La}_{2} \mathrm{Zr}_{2} \mathrm{O}_{7}$ ultrafine powders. Ceramics International. 42 , (5), 6221-6227 (2016).

23. Huang, Z., Duan, H., Liu, J., Zhang, H. Preparation of lanthanum cerate powders via a simple molten salt route. Ceramics International. 42 , (8), 10482-10486 (2016).

24. Wang, G., et al. Fabrication of rod-like $\mathrm{Ti}_{4} \mathrm{O}_{7}$ with high conductivity by molten salt synthesis. Materials Letters. 186,361-363 (2017).

25. Pokhrel, M., Burger, A., Groza, M., Mao, Y. Enhance the photoluminescence and radioluminescence of $\mathrm{La}_{2} \mathrm{Zr}_{2} \mathrm{O}_{7}: \mathrm{Eu}^{3+}$ core nanoparticles by coating with a thin $\mathrm{Y}_{2} \mathrm{O}_{3}$ shell. Optical Materials. 68 ,35-41 (2017).

26. Ramesh, G., Subramanian, V., Sivasubramanian, V. Dielectric properties of lead indium niobate ceramics synthesized by conventional solid state reaction method. Materials Research Bulletin. 45 , (12), 1871-1874 (2010).

27. Gupta, S. K., et al. Role of various defects in the photoluminescence characteristics of nanocrystalline $\mathrm{Nd}_{2} \mathrm{Zr}_{2} \mathrm{O}_{7}$ : $\mathrm{An}$ introspection through spectroscopic and DFT calculations. Journal of Materials Chemistry C. 4 , (22), 4988-5000 (2016).

28. Wang, X., Zhu, Y., Zhang, W. Preparation of lanthanum zirconate nano-powders by Molten Salt method. Journal of Non-Crystalline Solids. $356,(20-22), 1049-1051(2010)$

29. Popov, V. V., et al. Fluorite-pyrochlore phase transition in nanostructured $\mathrm{Ln}_{2} \mathrm{Hf}_{2} \mathrm{O}_{7}(\mathrm{Ln}=\mathrm{La}-\mathrm{Lu})$. Journal of Alloys and Compounds. 689 ,669-679 (2016).

30. Rybarczyk, M. K., Gontarek, E., Lieder, M., Titirici, M.-M. Salt melt synthesis of curved nitrogen-doped carbon nanostructures: ORR kinetics boost. Applied Surface Science. $435,543-551$ (2018).

31. Ozen, M., Mertens, M., Snikers, F., D'Hondt, H., Cool, P. Molten-salt synthesis of tetragonal micron-sized barium titanate from a peroxohydroxide precursor. Advanced Powder Technology. 28 , (1), 146-154 (2017).

32. Fazli, R., Fazli, M., Safaei-Naeini, Y., Golestani-Fard, F. The effects of processing parameters on formation of nano-spinel $\left(\mathrm{MgAl}_{2} \mathrm{O}_{4}\right)$ from $\mathrm{LiCl}$ molten salt. Ceramics International. 39 , (6), 6265-6270 (2013).

33. Bortolani, F., Dorey, R. A. Molten salt synthesis of PZT powder for direct write inks. Journal of the European Ceramic Society. $\mathbf{3 0}$, (10), 2073-2079 (2010).

34. Zhou, H., Mao, Y., Wong, S. S. Probing structure-parameter correlations in the molten synthesis of BaZrO ${ }_{3}$ perovskite submicron-sized particles. Chemistry of Materials. 19 , (22), 5238-5249 (2007).

35. Kimura, T., Machida, M., Yamaguchi, T., Newnham, R. E. Products of Reaction Between $\mathrm{PbO}$ and $\mathrm{Nb}_{2} \mathrm{O}_{5}$ in $\mathrm{Molten} \mathrm{KCl}_{\text {or }} \mathrm{NaCl}$. Journal of the American Ceramic Society. 66 , (10), 195-197 (1983).

36. Liu, S., et al. A novel rechargeable zinc-air battery with molten salt electrolyte. Journal of Power Sources. 342,435-441 (2017).

37. Huang, Z., Li, B., Liu, J. Molten-Salt Synthesis of Oxyapatite $\mathrm{La}_{9.33} \mathrm{Si}_{6} \mathrm{O}_{26}$ Powders as Electrolytes for Intermediate Temperature Solid Oxide Fuel Cells. Physica status solidi A - Applicationand Materials Science. 207 , (10), 2247-2251 (2010).

38. Ahmed, J., Mao, Y. Synthesis, characterization and electrocatalytic properties of delafossite $\mathrm{CuGaO}_{2}$. Journal of Solid State Chemistry. 242 , (1), 77-85 (2016).

39. Ahmed, J., Mao, Y. Ultrafine Iridium Oxide Nanorods Synthesized by Molten Salt Method toward Electrocatalytic Oxygen and Hydrogen Evolution Reactions. Electrochimica Acta. 212 ,686-693 (2016). 